Article

\title{
Dimensionality Reduction Differentiates Sensitive Force-Time Characteristics from Loaded and Unloaded Conditions throughout Competitive Military Training
}

\author{
Justin J. Merrigan * ${ }^{\mathbb{D}}$, Jason D. Stone, Jad Ramadan, Joshua A. Hagen $(\mathbb{D}$ and Andrew G. Thompson \\ Human Performance Innovation Center, Rockefeller Neuroscience Institute, West Virginia University, \\ Morgantown, WV 26505, USA; jason.stone1@hsc.wvu.edu (J.D.S.); jramadan@hsc.wvu.edu (J.R.); \\ joshua.hagen@hsc.wvu.edu (J.A.H.); andrewg.thompson@hsc.wvu.edu (A.G.T.) \\ * Correspondence: justin.merrigan@hsc.wvu.edu
}

Citation: Merrigan, J.J.; Stone, J.D.; Ramadan, J.; Hagen, J.A.; Thompson, A.G. Dimensionality Reduction Differentiates Sensitive Force-Time Characteristics from Loaded and Unloaded Conditions throughout Competitive Military Training. Sustainability 2021, 13, 6105. https:// doi.org/10.3390/su13116105

Academic Editor: Gianpiero Greco

Received: 3 May 2021

Accepted: 25 May 2021

Published: 28 May 2021

Publisher's Note: MDPI stays neutral with regard to jurisdictional claims in published maps and institutional affiliations.

Copyright: (C) 2021 by the authors. Licensee MDPI, Basel, Switzerland. This article is an open access article distributed under the terms and conditions of the Creative Commons Attribution (CC BY) license (https:// creativecommons.org/licenses/by/ $4.0 /)$.

\begin{abstract}
The purpose was to evaluate neuromuscular fatigue's effect on unloaded and loaded countermovement jump (CMJ) force-time characteristics during high-intensity tactical training. Eighteen male and sixteen female Marines completed two maximal effort CMJs, in unloaded (PVC pipe) and loaded (10 kg weight vest and $20 \mathrm{~kg}$ barbell) conditions, prior to, and 24, 48, and $72 \mathrm{~h}$ after starting the 4-day event. The top three variables from three principal components (PC) were analyzed using mixed-effects modeling (PC1-concentric mean power, eccentric peak force, and modified reactive strength index; PC2 - countermovement depth, eccentric mean power, and eccentric mean velocity; PC3 - braking duration, jump height, peak power). Metrics from PC1 and PC3 were reduced across training and from both loading conditions. Metrics from PC2 were similarly affected by external loading but were less influenced by training-induced fatigue. Jump performances with the barbell and with shallower countermovement depths did not change throughout training. Thus, $20 \mathrm{~kg}$ loaded CMJs are stable neuromuscular measures suitable for tracking chronic training adaptations. Monitoring unloaded and $10 \mathrm{~kg}$ loaded CMJ performances, along with movement strategies (i.e., countermovement rates and depth), may help identify moments of accumulated fatigue to inform training and recovery adjustments and improve the sustainability of personnel.
\end{abstract}

Keywords: weighted jump; jump squat; countermovement jump; tactical; Marines; neuromuscular fatigue; force plate(s); principal component analysis; PCA

\section{Introduction}

Military physical readiness training is intended to adequately prepare military personnel for the physical demands of various occupational tasks (e.g., endurance marches across rugged terrain, swift maneuvers under fire, jumping over and landing from obstacles, parachuting), which may be performed for long durations while bearing required equipment (i.e., external loads) [1,2]. The implementation of high training volumes and intensities to reach the required levels of various muscular fitness domains (e.g., strength, power, and endurance) may result in overaccumulations of neuromuscular fatigue (NMF) and, consequentially, high incidences of musculoskeletal injuries (MSKI) [3-6]. In active operational forces and schoolhouse trainees, physical training may account for up to $80 \%$ of sustained MSKI, which occur most commonly to the ankles, knees, and lumbar spine in the form of muscle sprains, joint strains, or stress fractures [7-11]. The consequences of high MSKI rates persist through increased financial burdens and restricted operational capacities $[10,12]$. Due to the influence of physical training-induced NMF on injury risks $[3,13]$, objective monitoring of NMF indicative metrics throughout preparatory training periods assists in confirming whether or not training regimens are eliciting positive (i.e., enhanced physical capacities) or negative (i.e., poor movement mechanics, heightened injury risk) adaptations over time [14]. 
The unloaded countermovement jump (CMJ) is a commonly used, reliable, and valid neuromuscular assessment of lower body power output in military populations [1,15-22]. Unloaded CMJ performance is related to military task performances [23], and empirical evidence supports its use as an appropriate means of detecting NMF following high-intensity military simulated tasks [17]. Due to greater accessibility and more controlled data collection environments, the majority of research examining NMF and CMJs has been conducted in sport settings. These studies revealed a number of reliable force-time characteristics (e.g., reactive strength index, relative eccentric and concentric power output, mean and peak forces) that influence CMJ performance, which is hindered in the face of NMF [14,24,25]. Reliable metrics derived from force plates during CMJ testing provide extensive context, at the individual level, with respect to neuromuscular strategies utilized to attain a certain jump height (e.g., performance outcome), thus are informative for training programs. Yet, despite the influence of jumping strategies on jump outcomes, such as the countermovement depth and velocity [26-29], additional research is necessary to more accurately identify the most suitable force-time metrics for detecting NMF $[14,24,25]$. The CMJ is more predictive of NMF after controlling for concentric duration, force, and power [24], but jump height may also return to the baseline while the effects of NMF on movement strategies (e.g., eccentric and concentric durations) persist [25]. Thus, considering the amount of force-time metrics available and the limited but emerging evidence of their usefulness for detecting NMF, it is pertinent to investigate the force-time metrics most responsive to NMF. These metrics should also be investigated within the specific military populations of interest, as physical demands vary significantly based on military occupational specialty (MOS).

Similar to biomechanical consequences of NMF, the influence of persistent external load requirements (i.e., combat and protective equipment) on biomechanical strategies (i.e., movement competency) represent another factor to consider when implementing force plate assessments. In military personnel, the addition of external load has shown decreased performances in jumping tasks [22,30] and high-intensity occupational-related duties (i.e., combative movements) [19,31,32], as well as increased injury risks [33]. Additionally, loaded CMJ performances were more associated with loaded combat maneuvers (combat dress uniform, gear, and assault rifle replica) than unloaded CMJ performances [22]. As such, the loaded and unloaded CMJ likely test different but complementary physical capabilities, as the ability to perform loaded $\mathrm{CMJ}$ is heavily influenced by maximal muscular strength [34]. Nonetheless, military personnel alter movement strategies and force production during jumping tasks as a result of external load carriage [35,36]. To jump higher under load or fatigue, military personnel may pursue a shallower countermovement depth or may apply forces through a longer concentric phase to obtain a faster propulsive velocity at take-off [37]. Despite the influence of load on CMJ force-time characteristics, it is not understood whether loading parameters may respond differently to NMF during competitive military training events. Therefore, the purpose of this study was to evaluate the change in unloaded and loaded CMJ force-time characteristics across a competitive high-intensity training event in active-duty Marines. We believe these findings may provide practitioners valuable insight regarding the proper implementation of force plate testing to inform their training program prescriptions and improve the sustainability of tactical personnel.

\section{Materials and Methods}

\subsection{Subjects}

The study sample comprised 18 male Marines (height, $178.2 \pm 3.6 \mathrm{~cm}$; body mass, $84.3 \pm 5.9 \mathrm{~kg}$ ) and 16 female Marines (height, $161.1 \pm 5.9 \mathrm{~cm}$; body mass, $58.1 \pm 16.5 \mathrm{~kg}$ ). 


\subsection{Procedures}

A prospective design was employed on CMJ testing during the United States Marine Corps' (USMC) 2019 High-intensity Tactical Training (HITT) Championship event, hosted at the Marine Corps Base in Quantico, Virginia [38]. The events were strategically created by USMC HITT instructors, a cadre from relevant schoolhouses (e.g., infantry), and academic subject matter experts to encapsulate all physiological systems necessary for duty. The HITT events were conducted across four successive days that comprised elements of marksmanship simulations performed under fatigue, weighted runs across rugged terrain, obstacle courses, combat fitness tests of strength and power, a combat swimmer challenge, pugil stick fighting, live-fire fitness challenges, a BeaverFit assault rig, and a HITT combine. Baseline force plate testing was conducted prior to any of the aforementioned events and was then replicated at 24,48 , and $72 \mathrm{~h}$ following the start of the HITT Championships. All force plate assessments were conducted between 5 and 6 a.m. Just outside of the testing room and prior to assessments, all of the Marines completed a brief (10-min) dynamic warm-up that was designed and led by the same HITT instructor each morning. The warm-up included $5 \mathrm{~min}$ of shuttle runs at an incrementally faster pace, followed by $5 \mathrm{~min}$ of dynamic stretching to replicate movement patterns similar to the upcoming field event (e.g., arm circles/swings, pushups, squatting/jumping/lunging, trunk twists, and etcetera). Following the dynamic warm-up, the Marines returned inside to perform two warm-up CMJs at $50 \%$ and $75 \%$ effort, followed by two maximal effort CMJs. The CMJs were executed under the following conditions: (1) unloaded with a polyvinyl chloride (PVC) pipe, (2) loaded with a $10 \mathrm{~kg}$ tactical fitness weighted vest, and (3) loaded with a $20 \mathrm{~kg}$ Eleiko barbell.

\subsection{Countermovement Jump (CMJ)}

Marines performed each jump, with no arm swing permitted, by starting in the standing tall position with hands grasping either a PVC pipe, a barbell placed across their shoulders, or the weighted vest's collar, depending on the testing condition. The force plates were zeroed, then Marines were instructed to stand as tall and as still as possible with each foot on one of the ForceDecks FD4000 Dual Force Platforms (Vald Performance, Brisbane, Queensland, Australia) to determine bodyweight. The force-time curve was visually inspected to ensure limited movement occurred in the weighing phase, and a signal was provided by the software when an accurate bodyweight measure was taken. The subject was then instructed to start with equal weight distribution on both force cells. Following a "3-2-1" countdown, Marines were instructed to drop into a self-selected countermovement depth and perform a maximal effort vertical jump "as quickly and explosively as possible" before landing back onto the force plates and returning to the starting position. For each jump, verbal encouragement was provided by HITT instructors and fellow Marines to ensure that maximal effort was given during each attempt. Force plate data were sampled at $1000 \mathrm{~Hz}$ and analyzed via ForceDecks software (Vald Performance, Brisbane, Queensland, Australia). This software detects the initiation of movement as a $30 \mathrm{~N}$ deviation from the initial bodyweight calculation, the eccentric to concentric phase moment as the lowest center of mass displacement, and the take-off as the moment in which the vertical forces fall $30 \mathrm{~N}$ below body mass. All phases and force-time metrics are defined in the ForceDecks user manual and prior literature [14,39-41]. Metrics included in the current manuscript were reliable according to prior findings [42].

\subsection{Statistical Analysis}

All statistical procedures were conducted in R version 3.6.2 (R Foundation, Vienna, Austria, https: / / www.R-project.org (accessed on 20 May 2020)) with the critical rejection region set to $p<0.05$. To control for body mass differences between individuals, all forcedriven metrics were scaled to the individual's body mass (i.e., mean concentric force divided by body mass). To start, a principal component analysis (PCA) was conducted, using the "FactoMineR" package (function PCA), to reduce the dimensionality of the force- 
time metrics for assessing the effects of load and fatigue on jump performances. Prior to the PCA, a measure of sampling adequacy (MSA) was checked for each measure and the entire data set using the Kaiser-Meyer-Olkin measure with the following cut points: $<50$, unacceptable; $0.50-0.60$, miserable; $0.60-0.70$, mediocre; $0.70-0.80$, middling; $0.80-0.90$, meritorious; and $>0.90$, marvelous [43]. Overall, the MSA was meritorious, at 0.83 , and thus, the PCA was used. It was decided to remove all variables that were mediocre or less to improve the analysis as these may be considered inappropriate for factor analyses or, at the least, doing so strengthens the PCA results [43]. The PCA was conducted using the correlational method (scale $=$ TRUE) since the force-time metrics included different scales (i.e., large force-domain values and small time-domain values). A scree plot was created, and the dimensions that explained the most variance were selected to determine the number of principal components (PC) used in the analysis [44]. Based on the Kaiser criterion, only PCs with eigenvalues $>1$ were included [45]. To aid in the feature reduction process, the squared cosine $\left(\cos ^{2}\right)$ was calculated (and listed) per metric within each PC. The $\cos ^{2}$ value can be interpreted as a variable importance score [46], as larger values indicated a larger contribution to the respective component. The size of the loading (in a PC biplot) is dependent upon this value.

Following a reduction of metrics, several linear mixed-effects models were run to determine the influence of external load and fatigue on force-time metrics during the CMJ. The mixed-effects modeling approaches were conducted to account for individual responses to the data over time (Level 1, pre, $24 \mathrm{~h}$ post, $48 \mathrm{~h}$ post, $72 \mathrm{~h}$ post) nested within individuals (Level 2), using the "nlme" package [47]. Dimensionality reduction of the data (PCA) left the following dependent variables included in the models: modified reactive strength index ( $\mathrm{RSI}_{\text {mod }}$ ), eccentric peak force, concentric mean power, countermovement depth, eccentric peak velocity, eccentric mean power, braking phase duration, jump height, and concentric peak power. The explanatory predictor variables in each model were the loading condition (PVC, weighted vest, and barbell) and time (pre, 24, 48, $72 \mathrm{~h}$ post start). Countermovement depth was also included as another predictor to understand its influence on force-time metrics of interest, as well as responses to training-induced fatigue and external loading. Since male and female Marines responded similarly to the external loading conditions and across time points, additional interdependent samples $t$-tests were conducted to evaluate gender differences on force-time metrics. Additionally, effect sizes and their respective $95 \%$ confidence intervals were calculated for each pairwise comparison of main effects.

\section{Results}

A scree plot and the Kaiser criterion identified 3 main PCs contributing to the overall variance in the model (eigenvalues: PC1 $=13.95$, PC2 $=3.72$, PC3 $=3.22$ ). Principal component 1,2 , and 3 , explained $60.7 \%, 16.2 \%$, and $14.00 \%$ of the variance in CMJ performances for a cumulative $90.9 \%$ of the overall model variance. PC1 was most strongly driven by the concentric mean force and power, eccentric peak force, and RSI mod (Table 1). Further, external loads $\left(R^{2}=0.141 ; p<0.001\right)$ and time $\left(R^{2}=0.024 ; p=0.02\right)$ were significant qualitative factors. Significant loadings were noted in PC1 for external loading conditions of unloaded $\left(\right.$ estimate $\left.=1.373 ; \cos ^{2}=0.893 ; p<0.001\right)$, vest (estimate $\left.=0.553 ; \cos ^{2}=0.930 ; p=0.038\right)$, and barbell (estimate $=-1.926 ; \cos ^{2}=0.917 ; p<0.001$ ), as well as the baseline time point (estimate $=0.976 ; \cos ^{2}=0.852 ; p=0.002$ ). 
Table 1. The loadings of all of metrics for each principal component (PC).

\begin{tabular}{|c|c|c|c|c|c|}
\hline \multicolumn{2}{|l|}{ PC1 } & \multicolumn{2}{|l|}{ PC2 } & \multicolumn{2}{|l|}{ PC3 } \\
\hline Metric & $\cos ^{2}$ & Metric & $\cos ^{2}$ & Metric & $\cos ^{2}$ \\
\hline $\mathrm{RSI}_{\mathrm{mod}}$ & 0.84 & Countermovement Dip & 0.69 & Braking Duration & 0.35 \\
\hline Eccentric Peak Force & 0.84 & Eccentric Mean Power & 0.54 & Concentric Peak Velocity & 0.34 \\
\hline Concentric Mean Power & 0.82 & Eccentric Peak Velocity & 0.42 & Jump Height & 0.33 \\
\hline Concentric Mean Force & 0.8 & Deceleration Impulse & 0.42 & Peak Power & 0.31 \\
\hline Concentric Peak Force & 0.78 & Eccentric Peak Power & 0.29 & Concentric Impulse & 0.3 \\
\hline Deceleration Mean Force & 0.77 & Concentric Duration & 0.29 & Braking Mean Force & 0.23 \\
\hline Braking RFD & 0.75 & Deceleration Duration & 0.21 & Deceleration Duration & 0.23 \\
\hline Deceleration RFD & 0.72 & Concentric RPD & 0.21 & Braking RFD & 0.17 \\
\hline Concentric RPD & 0.69 & Braking Duration & 0.14 & Deceleration Impulse & 0.16 \\
\hline Concentric Impulse & 0.67 & Braking Impulse & 0.14 & Countermovement Dip & 0.14 \\
\hline
\end{tabular}

RFD—rate of force development; RPD—rate of power development; $\cos ^{2}$ —highlights the importance of the metric in the PC based on its contribution to the squared distance of the observation to the origin.

Figure 1 displays the loadings for all force-time metrics with a $\cos ^{2}$ above 0.80 in bi-plots of PC1 and PC2, as well as PC1 and PC3, to visually present groupings of related variables and their PC loadings. PC2 was most strongly influenced by the countermovement depth, deceleration impulse, eccentric mean power, and eccentric peak velocity (Table 1). External load contributed to the loading of PC2 $\left(\mathrm{R}^{2}=0.046 ; p<0.001\right)$. Significant supplementary loadings were PVC, (estimate $\left.=-0.444 ; \cos ^{2}=0.094 ; p=0.002\right)$, barbell, (estimate $\left.=0.549 ; \cos ^{2}=0.075 ; p<0.001\right)$, as well as T72, (estimate $=0.363 ; \cos ^{2}=0.464$; $p=0.035$; Table 2, Figure 2). The third and final PC was most influenced by the braking duration, jump height, concentric peak velocity, and peak power (Table 1).

Table 2. Main effects and interactions of all mixed-effects models.

\begin{tabular}{|c|c|c|c|}
\hline \multicolumn{4}{|c|}{ Principal Component 1} \\
\hline & $\mathrm{RSI}_{\text {mod }}$ & Ecc. Peak Force & Con. Mean Power \\
\hline (Intercept) & $309.050 ;<0.001$ & $2632.683 ;<0.001$ & $649.137 ;<0.001$ \\
\hline Test Date & $14.371 ;<0.001$ & $29.884 ;<0.001$ & $23.586 ;<0.001$ \\
\hline External Load & $224.634 ;<0.001$ & $70.978 ;<0.001$ & $157.693 ;<0.001$ \\
\hline CM Depth & $0.855 ; 0.356$ & $27.767 ;<0.001$ & $6.039 ; 0.015$ \\
\hline Test Date ${ }^{*}$ Load & $2.937 ; 0.008$ & $0.614 ; 0.719$ & $1.516 ; 0.172$ \\
\hline Test Date * CM Depth & $3.151 ; 0.025$ & $0.801 ; 0.494$ & $2.906 ; 0.035$ \\
\hline Load * CM Depth & $0.373 ; 0.689$ & $0.573 ; 0.564$ & $0.398 ; 0.672$ \\
\hline \multicolumn{4}{|c|}{ Principal Component 2} \\
\hline & CM Depth & Ecc. Peak Velocity & Ecc. Mean Power \\
\hline (Intercept) & $1066.062 ;<0.001$ & $2371.727 ;<0.001$ & $3113.246 ;<0.001$ \\
\hline Test Date & $6.473 ;<0.001$ & $11.978 ;<0.001$ & $10.206 ;<0.001$ \\
\hline External Load & $5.392 ; 0.005$ & $21.696 ;<0.001$ & $23.838 ;<0.001$ \\
\hline CM Depth & - & $348.793 ;<0.001$ & $572.712 ;<0.001$ \\
\hline Test Date * Load & $0.124 ; 0.993$ & $0.571 ; 0.753$ & $0.629 ; 0.707$ \\
\hline Test Date ${ }^{*}$ CM Depth & - & $1.029 ; 0.380$ & $0.752 ; 0.522$ \\
\hline Load * CM Depth & - & $0.199 ; 0.820$ & $0.897 ; 0.409$ \\
\hline \multicolumn{4}{|c|}{ Principal Component 3} \\
\hline & Braking Duration & Jump Height & Con. Peak Power \\
\hline (Intercept) & $1904.1311 ;<0.001$ & $399.406 ;<0.001$ & $754.483 ;<0.001$ \\
\hline Test Date & $7.497 ;<0.001$ & $22.442 ;<0.001$ & $10.503 ;<0.001$ \\
\hline External Load & $57.558 ;<0.001$ & $157.308 ;<0.001$ & $178.441 ;<0.001$ \\
\hline CM Depth & $52.551 ;<0.001$ & $32.699 ;<0.001$ & $4.573 ; 0.033$ \\
\hline Test Date ${ }^{*}$ Load & $1.094 ; 0.366$ & $3.140 ; 0.005$ & $1.310 ; 0.252$ \\
\hline
\end{tabular}


Table 2. Cont.

\begin{tabular}{|c|c|c|c|}
\hline Test Date * CM Depth & $0.726 ; 0.537$ & $5.371 ; 0.001$ & $2.352 ; 0.072$ \\
\hline Load * CM Depth & $1.712 ; 0.182$ & $0.430 ; 0.651$ & $0.086 ; 0.917$ \\
\hline \multicolumn{4}{|c|}{$\begin{array}{l}\text { Results are presented as the } F \text {-value; } p \text {-value. }{ }^{*} \text {, indicates a significant explanatory variable of the following } \\
\text { meanings: Test Date is the main effect of time across the } 4 \text { testing dates; External Load is the main effect of loading } \\
\text { conditions (PVC, } 10 \mathrm{~kg} \text { weighted vest, } 20 \mathrm{~kg} \text { barbell); CM depth, is the main effect of countermovement depth on } \\
\text { the metric outcome; Test Date }{ }^{*} \text { Load is the interaction of time and external loading conditions; Test Date }{ }^{*} \mathrm{CM} \\
\text { Depth is the interaction effect of CM Depth on changes over time; Load }{ }^{*} \mathrm{CM} \text { Depth is the interaction effect of } \\
\text { CM Depth on external load effects on each metric. CM-countermovement; RSImod-modified reactive strength } \\
\text { index; Con.- concentric; Ecc. - eccentric. Peak concentric velocity was not used as it is a metric used to calculate } \\
\text { impulse-momentum-derived jump height and followed the same trend in change over time. }\end{array}$} \\
\hline
\end{tabular}

A

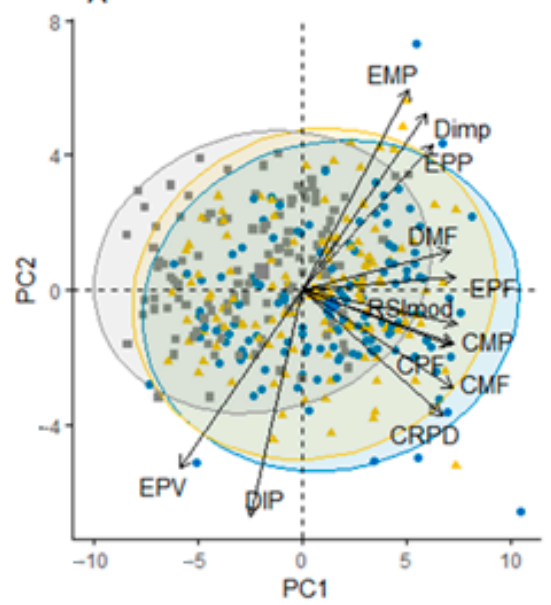

C

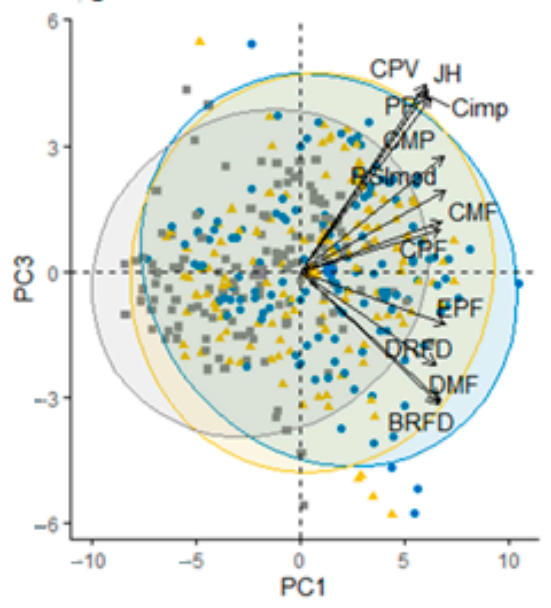

B

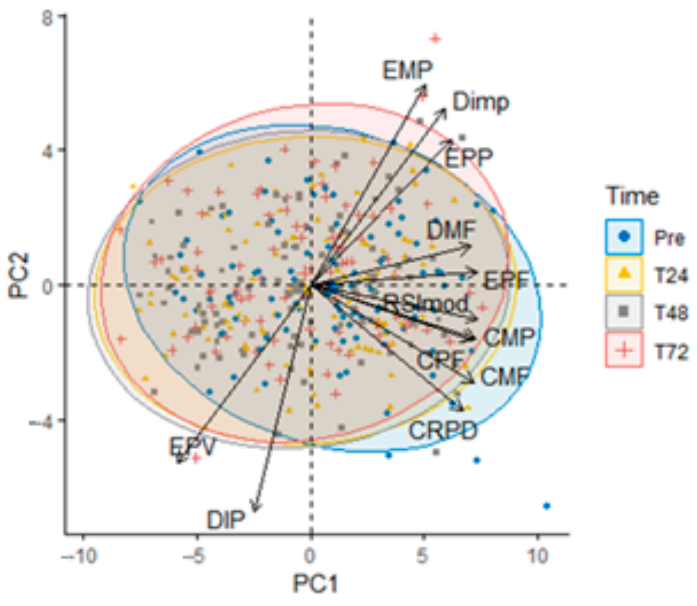

D

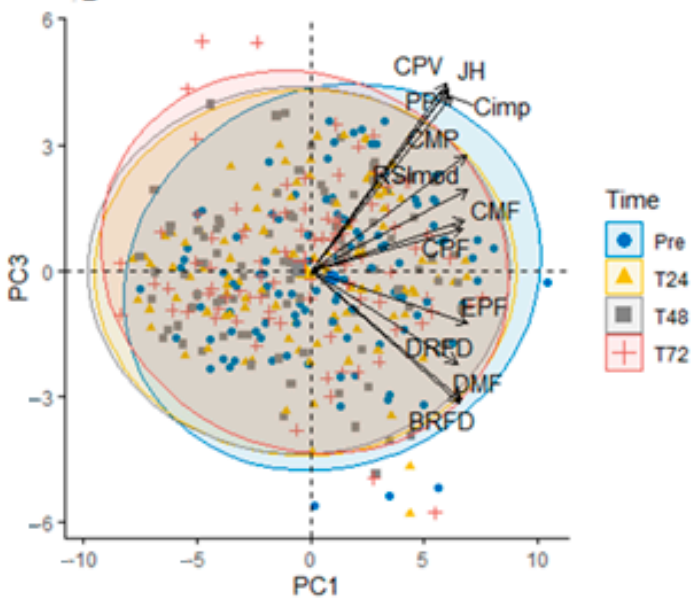

Figure 1. Loadings ( $\left.\cos ^{2} \geq 0.80\right)$ and correlations of force-time metrics and qualitative factors for principal components 1 and 2 (loading conditions, (A); time, (B)), as well as principal components 1 and 3 (loading conditions, (C); time, (D)). Metric loadings (arrows) appearing closely grouped together are strongly correlated with each other; thus, providing similar information. Metric abbreviations are as follows: EMP_eccentric mean force; Dimp—deceleration impulse; EPPeccentric peak power; DMF—deceleration mean force: EPF_eccentric peak force; modRSI-modified reactive strength index; $\mathrm{CMP}$ - concentric mean power; $\mathrm{CPF}$ — concentric peak force; $\mathrm{CMF}$ - concentric mean force; CRPD—concentric rate of power development; CMdepth—countermovement depth; EPV—eccentric peak velocity; CPV—concentric peak velocity; JH—jump height; PP—peak power; Cimp—concentric impulse; DRFD—deceleration rate of force development; BRFD—braking rate of force development. 

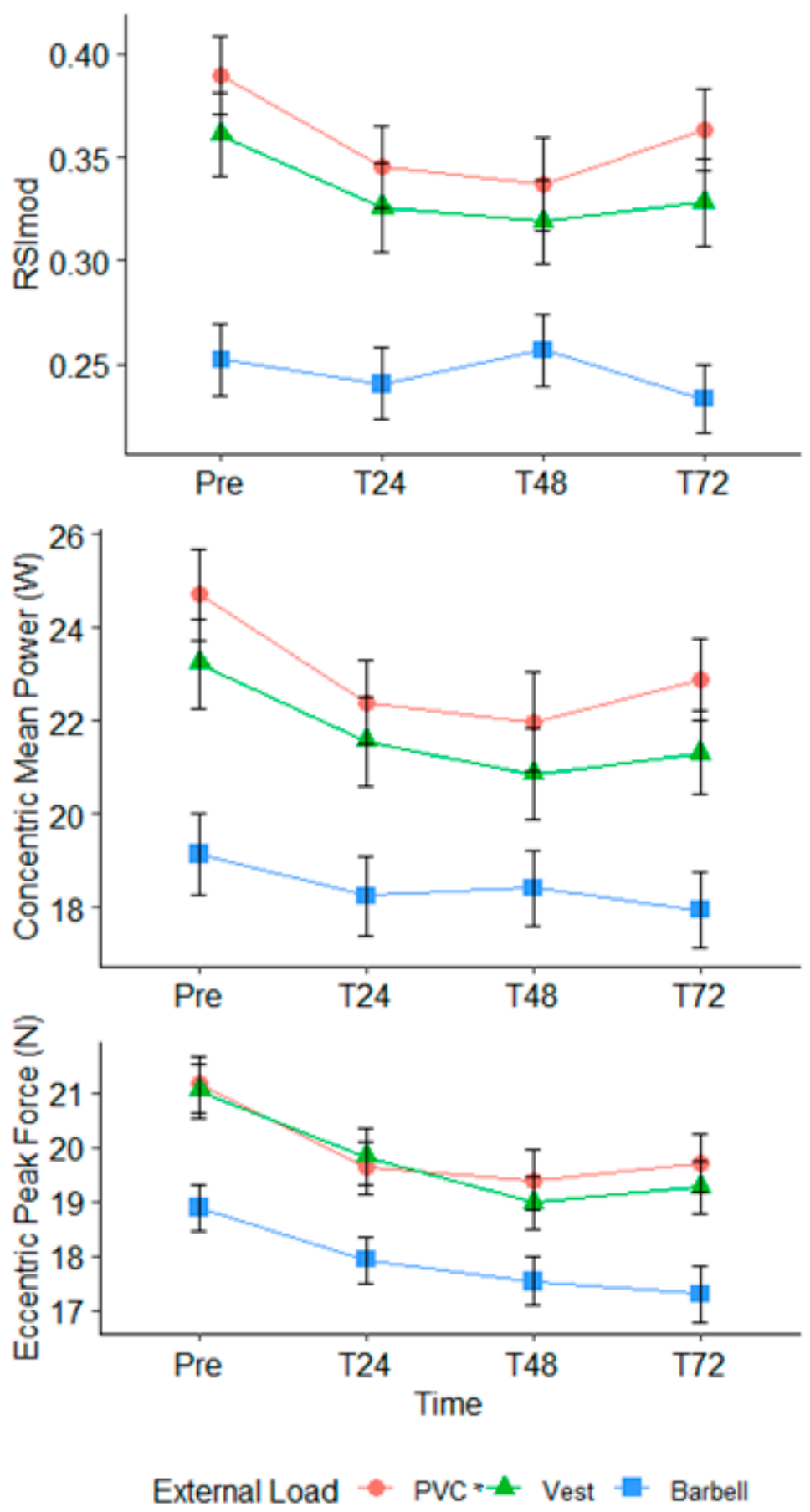

Figure 2. Changes to the top three contributors for principal component 1, including modified reactive strength index (RSImod), concentric mean power, and eccentric peak force over the course of the high-intensity training event under each condition (unloaded, PVC; loaded with a $10 \mathrm{~kg}$ vest; and loaded with $20 \mathrm{~kg}$, barbell). *, indicates a significant difference from baseline for respective conditions (PVC and vest). Labels of PVC and vest within the figure indicate significant differences in the unloaded PVC and $10 \mathrm{~kg}$ weighted vest conditions, respectively.

The top 3 variables that most contributed to each PC (as determined by $\cos ^{2}$, see Table 1) were included in linear mixed-effects models to investigate the effects of external load and fatigue (Table 2). There were significant main effects of time and external load for all selected force-time metrics, while the interaction between time and external load was sig-

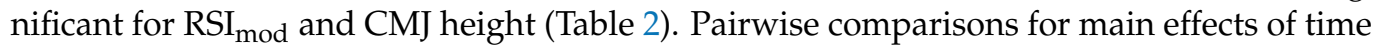
and external load are displayed in Tables 3 and 4, respectively. The data for each condition 
over time for metrics in PC2 are displayed in Figure 3. Effects of time on each condition

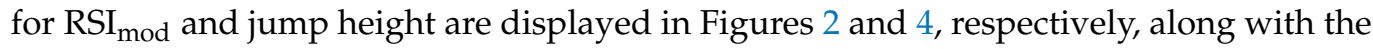
other 2 variables from PC1 and PC3. For every one-unit increase in countermovement depth, the following metrics were decreased: CMJ height $(-0.230 \pm 0.068 \mathrm{~cm})$, concentric power $(-0.085 \pm 0.047 \mathrm{~W})$, eccentric power $(-0.124 \pm 0.009 \mathrm{~W})$, eccentric peak velocity $\left(0.021 \pm 0.002 \mathrm{~m} \cdot \mathrm{s}^{-1}\right)$, and eccentric peak force $(-0.115 \pm 0.033 \mathrm{~N})$. The effect of external loading was not influenced by countermovement depth; however, shallower countermovement depths resulted in less change in $\mathrm{RSI}_{\text {mod, }}$, concentric mean power, and CMJ height (Table 2). Males demonstrated higher jump heights, concentric power output, and RSI mod than females (Table 5). Countermovement depth was deeper in males, but these differences were mitigated after accounting for differences in body height (Table 5).

Table 3. Pairwise comparisons of force-time metrics over time collapsed across conditions.

\begin{tabular}{|c|c|c|c|c|c|c|c|}
\hline \multirow[b]{2}{*}{ Metric } & \multicolumn{4}{|c|}{ Mean \pm SE } & \multicolumn{3}{|c|}{ Effect Size (95\% Confidence Intervals) } \\
\hline & Pre & T24 & T48 & T72 & (Pre-T24) & (Pre-T48) & (Pre-T72) \\
\hline $\begin{array}{l}\text { Eccentric Peak } \\
\text { Force (N) }\end{array}$ & $20.3 \pm 0.4$ & $19.2 \pm 0.4$ & $18.7 \pm 0.4$ & $18.5 \pm 0.4$ & $\begin{array}{c}0.73^{*} \\
(0.44,1.01)\end{array}$ & $\begin{array}{r}1.0 \\
(0.78\end{array}$ & $\begin{array}{r}1 . \\
(0.91\end{array}$ \\
\hline Peak Power (W) & $41.8 \pm 1.5$ & $40.0 \pm 1.5$ & $39.3 \pm 1.5$ & $39.5 \pm 1.5$ & $\begin{array}{r}0.4 \\
(0.19\end{array}$ & $\begin{array}{r}0.6 \\
(0.38\end{array}$ & $\begin{array}{r}0.6 \\
(0.33\end{array}$ \\
\hline $\begin{array}{c}\text { Braking } \\
\text { Duration (s) }\end{array}$ & $0.369 \pm 0.009$ & $0.390 \pm 0.009$ & $0.395 \pm 0.010$ & & $\begin{array}{c}-0.55^{*} \\
(-0.83,-0.26)\end{array}$ & $\begin{array}{c}-0.68^{*} \\
(-0.98,-0.39)\end{array}$ & $\begin{array}{c}-0.60 \text { * } \\
(-0.90,-0.31)\end{array}$ \\
\hline $\begin{array}{l}\text { Eccentric Mean } \\
\text { Power (W) }\end{array}$ & $6.01 \pm 0.11$ & $5.94 \pm 0.11$ & $5.94 \pm 0.11$ & $5.85 \pm 0.11$ & $\begin{array}{c}0.18 \\
(-0.10,0.47)\end{array}$ & $\begin{array}{c}0.20 \\
(-0.09,0.49)\end{array}$ & $\begin{array}{c}0.41 * \\
(0.11,0.70)\end{array}$ \\
\hline $\begin{array}{l}\text { Eccentric Peak } \\
\text { Velocity }\left(\mathrm{m} \cdot \mathrm{s}^{-1}\right)\end{array}$ & $-1.15 \pm 0.024$ & $-1.12 \pm 0.02$ & $-1.11 \pm 0.02$ & $-1.10 \pm 0.02$ & $\begin{array}{c}-0.33 \\
(-0.61,-0.05)\end{array}$ & $\begin{array}{c}-0.41 \text { * } \\
(-0.70,-0.11)\end{array}$ & $\begin{array}{c}-0.47 \text { * } \\
(-0.77,-0.17)\end{array}$ \\
\hline $\begin{array}{l}\text { Countermovement } \\
\text { Depth }(\mathrm{cm})\end{array}$ & $-34.0 \pm 1.07$ & $-32.7 \pm 1.1$ & $-32.4 \pm 1.1$ & $-34.5 \pm 1.1$ & $\begin{array}{c}-0.33 \\
(-0.61,-0.05)\end{array}$ & $\begin{array}{c}-0.41 * \\
(-0.70,-0.12)\end{array}$ & $\begin{array}{c}0.14 \\
(-0.15,0.43)\end{array}$ \\
\hline $\begin{array}{l}\text { Concentric Mean } \\
\text { Power (w) }\end{array}$ & $22.3 \pm 0.8$ & $20.8 \pm 0.8$ & $20.2 \pm 0.8$ & $20.1 \pm 0.8$ & $\begin{array}{c}0.71 * \\
(0.42,1.01)\end{array}$ & $\begin{array}{c}0.99 * \\
(0.69,1.29)\end{array}$ & $\begin{array}{c}1.03 * \\
(0.73,1.34)\end{array}$ \\
\hline Jump Height (cm) & $27.0 \pm 1.3$ & $24.8 \pm 1.3$ & $24.0 \pm 1.3$ & $24.4 \pm 1.3$ & $\begin{array}{c}0.70 * \\
(0.41,0.99)\end{array}$ & $\begin{array}{c}0.97 * \\
(0.67,1.28)\end{array}$ & $\begin{array}{c}0.83 * \\
(0.53,1.13)\end{array}$ \\
\hline $\begin{array}{c}\text { Modified } \\
\text { Reactive Strength } \\
\text { Index }\left(\mathrm{m} \cdot \mathrm{s}^{-1}\right)\end{array}$ & $0.334 \pm 0.018$ & $0.305 \pm 0.018$ & $0.300 \pm 0.018$ & $0.299 \pm 0.018$ & $\begin{array}{c}0.66^{*} \\
(0.37,0.95)\end{array}$ & $\begin{array}{c}0.77 * \\
(0.47,1.07)\end{array}$ & $\begin{array}{c}0.80 * \\
(0.50,1.10)\end{array}$ \\
\hline
\end{tabular}

*, indicates significant difference at $p<0.05$.

Table 4. Pairwise comparisons of force-time metrics across conditions collapsed across time.

\begin{tabular}{|c|c|c|c|c|c|c|}
\hline \multirow[b]{2}{*}{ Metric } & \multicolumn{3}{|c|}{ Mean \pm SE } & \multicolumn{3}{|c|}{ Effect Size (95\% Confidence Intervals) } \\
\hline & PVC Pipe & 10 kg Vest & $20 \mathrm{~kg} \mathrm{~B}$ & (Barbell-PVC) & (Barbell-Vest) & (PVC-Vest) \\
\hline $\begin{array}{l}\text { Eccentric Peak } \\
\text { Force (N) }\end{array}$ & $20.0 \pm 0.4$ & $19.7 \pm 0.4$ & $17.8 \pm 0.4$ & $\begin{array}{c}-1.49 \\
(-1.78,-1.21)\end{array}$ & $\begin{array}{c}-1.31 \\
(-1.58,-1.04) *\end{array}$ & $\begin{array}{c}0.179 \\
(-0.07,0.43)\end{array}$ \\
\hline Peak Power $(W)$ & $41.3 \pm 1.5$ & $43.9 \pm 1.5$ & $35.2 \pm 1.5$ & $\begin{array}{c}-2.37 \\
(-2.68,-2.06) *\end{array}$ & $\begin{array}{c}-1.672 \\
(-1.95,-1.39) *\end{array}$ & $\begin{array}{c}0.70 \\
(0.44,0.95) *\end{array}$ \\
\hline Braking Duration (s) & $0.367 \pm 0.009$ & $0.379 \pm 0.009$ & $0.413 \pm 0.009$ & $\begin{array}{c}1.19 \\
(0.92,1.46) *\end{array}$ & $\begin{array}{c}0.88 \\
(0.62,1.13) *\end{array}$ & $\begin{array}{c}-0.31 \\
(-0.56,-0.06)\end{array}$ \\
\hline $\begin{array}{l}\text { Eccentric Mean } \\
\text { Power (W) }\end{array}$ & $6.09 \pm 0.11$ & $6.08 \pm 0.11$ & & $\begin{array}{c}-1.16 \\
(-1.43,-0.89) *\end{array}$ & $\begin{array}{c}-1.14 \\
(-1.41,-0.87) *\end{array}$ & $\begin{array}{c}0.02 \\
(-0.23,0.27)\end{array}$ \\
\hline $\begin{array}{l}\text { Eccentric Peak } \\
\text { Velocity }\left(\mathrm{m} \cdot \mathrm{s}^{-1}\right)\end{array}$ & $-1.16 \pm 0.02$ & $-1.13 \pm 0.02$ & $-1.06 \pm 0.02$ & $\begin{array}{c}1.20 \\
(0.93,1.47) *\end{array}$ & $\begin{array}{c}0.86 \\
(0.60,1.12)\end{array}$ & $\begin{array}{c}-0.34 \\
(-0.59,-0.09)\end{array}$ \\
\hline $\begin{array}{l}\text { Countermovement } \\
\text { Depth }(\mathrm{cm})\end{array}$ & $-32.7 \pm 1.1$ & $-33.3 \pm 1.1$ & $-34.2 \pm 1.1$ & $\begin{array}{c}-0.41 \\
(-0.66,-0.16)\end{array}$ & $\begin{array}{c}-0.24 \\
(-0.49,0.01)\end{array}$ & $\begin{array}{c}0.817 \\
(-0.08,0.41)\end{array}$ \\
\hline $\begin{array}{l}\text { Concentric Mean } \\
\text { Power }(\mathrm{w})\end{array}$ & $22.9 \pm 0.8$ & $21.6 \pm 0.8$ & $18.2 \pm 0.8$ & $\begin{array}{c}-2.24 \\
(-2.54,-1.94) *\end{array}$ & 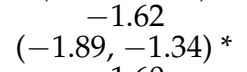 & $\begin{array}{c}0.625 \\
(0.37,0.88)\end{array}$ \\
\hline Jump Height (cm) & $28.1 \pm 1.3$ & $26.0 \pm 1.3$ & $21.0 \pm 1.3$ & $\begin{array}{c}-2.31 \\
(-2.61,-2.00) *\end{array}$ & $\begin{array}{c}-1.60 \\
(-1.88,-1.32) *\end{array}$ & $\begin{array}{c}0.71 \\
(0.45,0.96) *\end{array}$ \\
\hline $\begin{array}{l}\text { Modified Reactive } \\
\text { Strength Index }\left(\mathrm{m} \cdot \mathrm{s}^{-1}\right)\end{array}$ & $0.356 \pm 0.018$ & $0.330 \pm 0.0$ & $.242 \pm 0.0178$ & $\begin{array}{c}-2.58 \\
(-2.90,-2.26)\end{array}$ & $\begin{array}{c}-1.99 \\
(-2.28,-1.69)\end{array}$ & $\begin{array}{c}0.59 \\
(0.34,0.84) *\end{array}$ \\
\hline
\end{tabular}

$*$, indicates significant difference at $p<0.05$. 

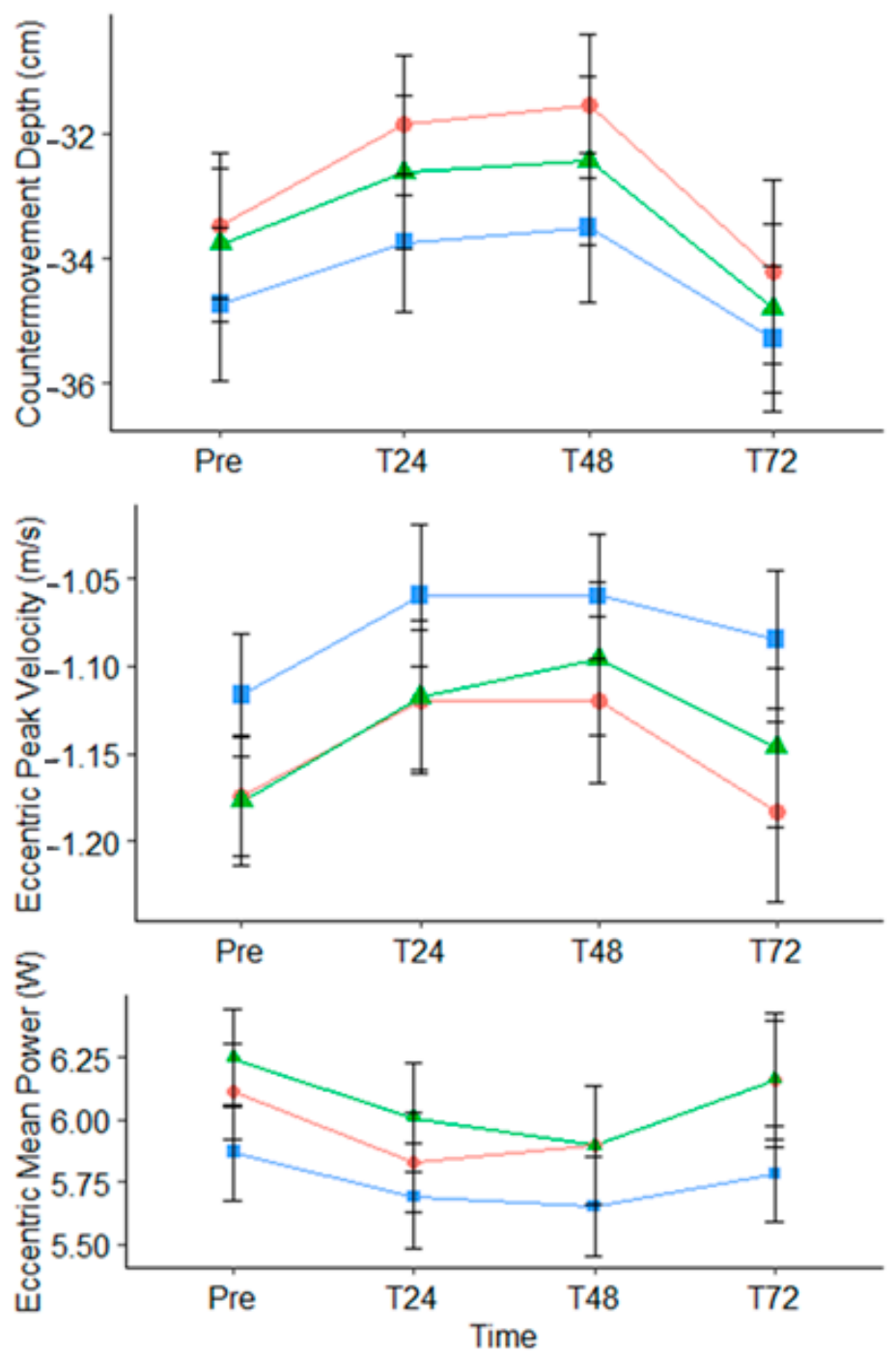

External Load $\rightarrow$ PVC $\_$Vest - Barbell

Figure 3. Changes to the top three contributors for principal component 2 over the course of the highintensity training event under each condition (unloaded, PVC; loaded with $10 \mathrm{~kg}$, vest; and loaded with $20 \mathrm{~kg}$, barbell).

Table 5. Descriptive Statistics and effect size comparisons between male and female Marines.

\begin{tabular}{|c|c|c|c|c|}
\hline Force-Time Metric & Female (16) & Male (18) & $t$-Stat & $\begin{array}{l}\text { Cohen's D } \\
(95 \% \text { CI })\end{array}$ \\
\hline Eccentric Peak Force (N) & $20.28 \pm 2.760$ & $21.94 \pm 3.01$ & -1.68 & $0.59(-0.12,1.29)$ \\
\hline Peak Power (W) & $38.04 \pm 4.55$ & $53.62 \pm 7.34$ & $-7.52 *$ & $2.59(1.44,3.72)$ \\
\hline Braking Duration (s) & $0.35 \pm 0.08$ & $0.36 \pm 0.06$ & -0.26 & $0.10(-0.58,0.77)$ \\
\hline Eccentric Mean Power (W) & $5.80 \pm 0.84$ & $2918 \pm 6.39$ & -1.59 & $0.55(-0.16,1.24)$ \\
\hline $\begin{array}{l}\text { Eccentric Peak Velocity } \\
\qquad\left(\mathrm{m} \cdot \mathrm{s}^{-1}\right)\end{array}$ & $-1.11 \pm 0.18$ & $-1.23 \pm 0.21$ & 1.91 & $-0.67(-1.38,0.05)$ \\
\hline $\begin{array}{l}\text { Countermovement, } \\
\text { CM Depth }(\mathrm{cm})\end{array}$ & $-30.31 \pm 4.53$ & $-36.28 \pm 7.36$ & $2.89 *$ & $0.99(-1.74,-0.22)$ \\
\hline CM Depth/Height $(\mathrm{cm} / \mathrm{cm})$ & $-0.19 \pm 0.03$ & $-0.20 \pm 0.04$ & 0.82 & $-1.00(-0.3,0.41)$ \\
\hline Height $(\mathrm{cm})$ & $161.1 \pm 5.9$ & $178.2 \pm 3.6$ & $-8.26 *$ & $3.02(1.73,4.28)$ \\
\hline Concentric Mean Power (w) & $20.39 \pm 3.41$ & $28.54 \pm 4.45$ & $-6.03 *$ & $2.10(1.08,3.09)$ \\
\hline Jump Height $(\mathrm{cm})$ & $23.19 \pm 3.73$ & $37.66 \pm 5.62$ & $-8.93 *$ & $3.09(1.79,4.36)$ \\
\hline $\begin{array}{l}\text { Modified Reactive Strength } \\
\text { Index }\left(\mathrm{m} \cdot \mathrm{s}^{-1}\right)\end{array}$ & $0.31 \pm 0.08$ & $0.46 \pm 0.08$ & $-5.48^{*}$ & $1.93(0.95,2.87)$ \\
\hline
\end{tabular}

${ }^{*}$, indicates statistically a significant difference between male and female Marines. 

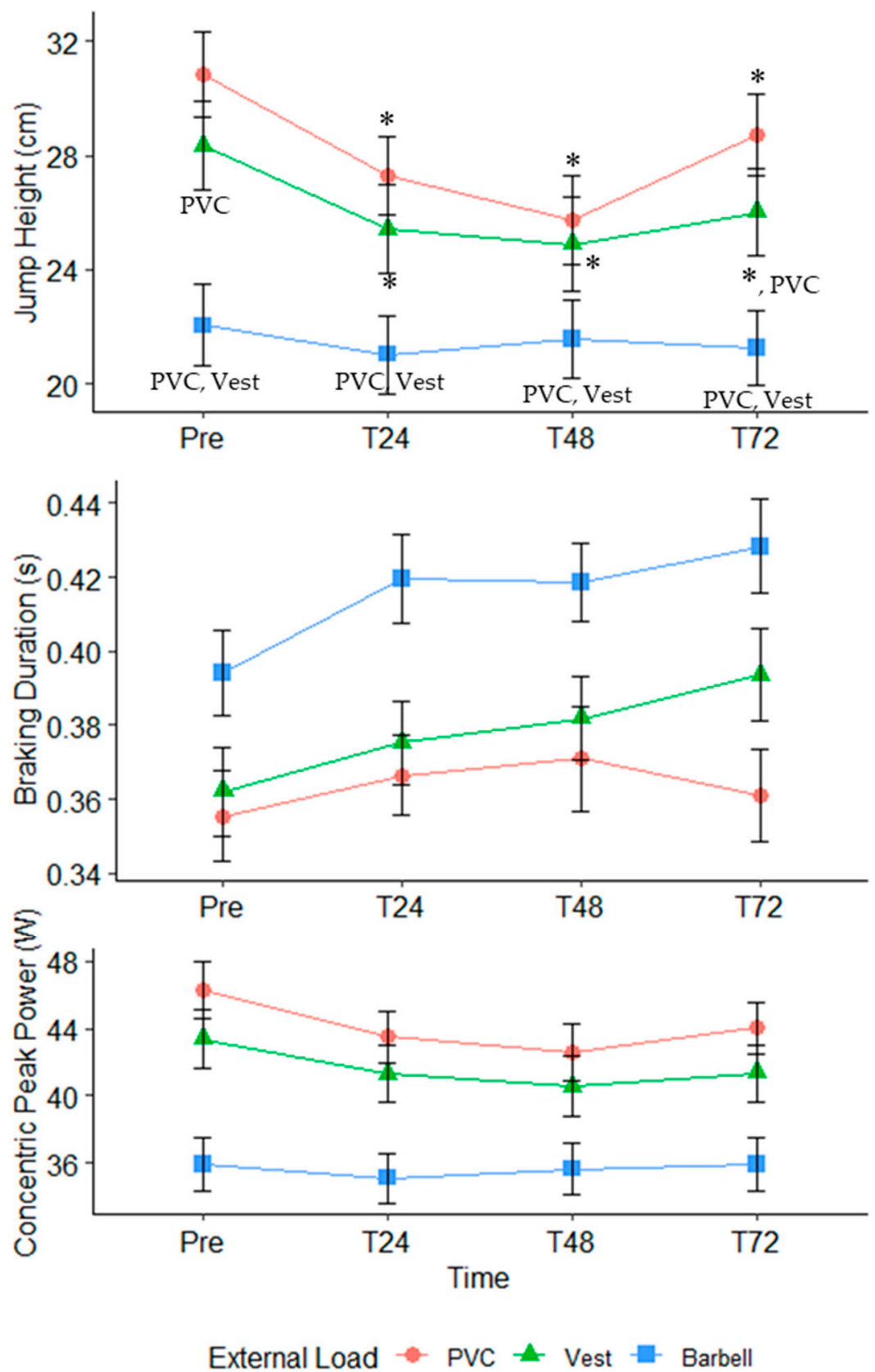

Figure 4. Changes to the top three contributors for principal component 3 over the course of the highintensity training event under each condition (unloaded, PVC; loaded with $10 \mathrm{~kg}$, vest; and loaded with $20 \mathrm{~kg}$, barbell). *, indicates a significant difference from baseline for respective conditions (PVC and vest). Labels of PVC and vest within the figure indicate significant differences in the unloaded PVC and $10 \mathrm{~kg}$ weighted vest conditions, respectively.

\section{Discussion}

To our knowledge, the current study is the first empirical effort to document the influence of load and fatigue and their interaction on numerous CMJ force-time characteristics across a competition training event in military personnel. The application of PCA was critical to identify a reduced number of uncorrelated force-time metrics that were most influential in explaining the variance in jump performances in two conditions, over time and under load. The PCA identified three PCs contributing to the variance in CMJ performances, making the force-time data more understandable and scalable for future implementation by practitioners and military personnel. One of the main findings 
was the change in the jumping strategy and decrease in performance while loaded with a $20 \mathrm{~kg}$ barbell, compared to the PVC pipe and $10 \mathrm{~kg}$ weighted vest. Further, the CMJ with a barbell did not change performances across the week; thus, was less sensitive to NMF than the PVC pipe and $10 \mathrm{~kg}$ weighted vest. Likewise, those who performed the jump with a shallower countermovement noted a slower and less powerful countermovement and propulsive ability (e.g., lower jump height) at baseline, which led to less of a change in jump height throughout the event. Thus, this study provided strategies for selecting the most important test and metrics for profiling and monitoring purposes during bouts of high-intensity tactical training.

The use of PCA can be implemented by scientists and practitioners as a means for reducing the amount of force-time metrics being used to monitor NMF in their personnel. Ultimately, this allows the practitioner to select the fewest number of variables to monitor based on their unique contributions to the variance within their dataset (i.e., individual differences or intraindividual differences from NMF). This technique, especially when performed with data collected from the specific population, allows for an in-depth and scalable analysis across any force plate technology. In the current study, the greatest contributor to PC1 was the ability to change from eccentric to concentric actions, which is often measured via the RSI mod. Additionally, peak forces during the eccentric phase and mean concentric forces and power were significant contributors to PC1, which may be described as the driving factors for jump height capability. The main contributors in PC2 described the strategy imposed to reach the outcome, such as countermovement depth, eccentric peak velocity, and eccentric mean power. The third PC may be described as the overall outcome, which is jump height. The other leading factors, being peak velocity and power, are likely telling the same story as jump height when considering that jump height and peak power are derived from peak velocity (impulse-momentum relationship) or linearly related with velocity [48]. Meanwhile, the braking phase duration may be an additional explanatory variable for jump height, as the braking phase duration often differentiates strategies and outcomes of high and low jump performers [49]. In summary, the results of the PCA would suggest that the majority of variance in jump performances were explained by the efficiency of the stretch-shortening cycle (i.e., ability to rapidly transfer energy from the eccentric to concentric phase), the strategies utilized throughout the jump, and the overall outcome itself (i.e., jump height). Thus, in military settings, monitoring jump performances with force plates should consist of at least one metric from each of these categories to help explain the variations in jumping ability.

The first PC was influenced by external loading and the baseline time point, suggesting that the loading conditions differed, and the baseline value was different from all other time points. The longitudinal models in the current study found a significant reduction in all force-time metrics of interest, which never returned to the baseline, while countermovement depth was reduced at $48 \mathrm{~h}$ and returned to the baseline by $72 \mathrm{~h}$ following the start of the events. Male collegiate team sport athletes demonstrated a return of mean power and peak velocity by $72 \mathrm{~h}$ following a fatiguing high-intensity interval running program [50]. In U.S. Marines, jump height and power were reduced following 8 days of sustained operations [17]. Likewise, in other military personnel, 21-day training resulted in decrements in standing long jump performances mid-training, immediately post-training, and following a 4-day recovery [51]. There were many considerations for the responses to high-intensity stretch-shortening cycle tasks. Namely, the intensity and sheer load-volume of the task drove the bimodal recovery response, which typically resulted in an immediate small increase, followed by a steady decline in performance for up to 4-8 days following the training [52]. Interestingly, the countermovement depth, eccentric velocity, and eccentric mean power did not follow the same patterns of change over the course of the training events as the performance outcomes. Thus, although strategies may be altered immediately after a fatiguing event [37], the responses over time may vary.

The novel finding from the current study was the impact of the load on the response of CMJ force-time characteristics to fatiguing training in Marines. Of note, the $10 \mathrm{~kg}$ 
weighted vest responded similarly to the unloaded jump performances over the course of the training events and, resultantly, would be a useful assessment for detecting NMF.

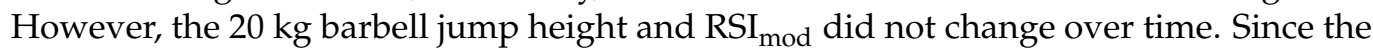
reductions in jump strategies and abilities undoubtedly increased as external loading magnitudes increased [53], the $20 \mathrm{~kg}$ barbell resulted in greater reductions of baseline jump performances, which may explain the greater stability in $20 \mathrm{~kg}$ loaded jump performances throughout the training events. The performance reductions were likely a result of altered jumping strategies, as the current sample of Marines executed the $20 \mathrm{~kg}$ loaded jump with a deeper countermovement depth, slower eccentric velocity, less powerful and forceful eccentric phase, and longer braking duration. Prior research in ROTC cadets suggested that greater heights of externally loaded jumps might be achieved when a shallower countermovement depth or longer acceleration phase duration was utilized [37]. It was also likely that the loaded jump with $20 \mathrm{~kg}$ was more of a measure of relative strength levels or explosive strength, which was a different construct than unloaded CMJ measures [34]. Similarly, when monitoring isometric-mid-thigh pull performances, the power measures (i.e., rate of force development) were more sensitive to NMF than strength measures (i.e., peak force) [54]. The loaded jumps with a $20 \mathrm{~kg}$ barbell were more indicative of relative strength capacities and movement competencies under load but were likely too stable for the detection of NMF throughout a brief intensive training bout. Therefore, it is recommended to use a $20 \mathrm{~kg}$ loaded jump to assess strength levels but, for monitoring NMF, unloaded or lightly loaded conditions are more appropriate, although more research is recommended over longer training durations.

Of note, the influence of the countermovement depth may suggest its versatility as an explanatory variable for jump performance capabilities between individuals and within individuals across different conditions. Prior literature on unloaded CMJs found the countermovement depth to be lower during unimodal force-time curves [49], and the shallower countermovement depth resulted in lower jump heights [27-29]. In agreement, as the current findings suggested, shallower countermovement depth resulted in a slower and less forceful and powerful eccentric phase, followed by a less powerful concentric phase and lower jump height. Those jumping with a shallow countermovement depth had less change in jump height, concentric mean power, and RSI mod from the events. This may be a result of the lower initial jump values, alike the loaded jumps with $20 \mathrm{~kg}$. For low jump performers, there may be less of a decline in jump abilities due to NMF compared to high jump performers. Nonetheless, the magnitude of the depth and rate of the countermovement were contributors to jump performances and responded to loading conditions and NMF; thus, should be included in monitoring programs to better explain differences between individuals and changes over time.

One limitation worth mentioning is that the $20 \mathrm{~kg}$ load was placed on the shoulder with a barbell compared to increasing the weighted vest. Shoulder load has shown to have more consequences on performance than loads placed at arm's length [55], but it is uncertain how the shoulder load of a barbell compares to a weighted vest. Thus, it was difficult to determine how much of the results of the current study stem from the load compared to the placement of the barbell. Future research should consider similar investigations using a spectrum of loads exclusively using weighted vests and/or barbells. Yet, the current findings may be used to develop concepts for monitoring neuromuscular fatigue and levels of physical preparedness during high-intensity training events prior to and during deployments. Lastly, it is important to note that male and female Marines responded similarly to the loading conditions and training-induced fatigue. However, females had lower jumping performances despite utilizing similar eccentric movement strategies, consistent with prior literature [30]. Thus, females should partake in resistance training aimed at an increased power output at various external loads to reduce the gender gap and improve physical preparedness for sustainable careers. 


\section{Conclusions}

According to a PCA, the variance of the CMJ performances due to fatigue and external loading was predominately explained by the ability to utilize the stretch-shortening cycle, followed by the strategy used to execute the jumps and the outcome of the jump itself (e.g., jump height). Although jump performances were reduced due to the $10 \mathrm{~kg}$ weighted vest, the $20 \mathrm{~kg}$ barbell resulted in greater reductions in jump performances compared to the unloaded condition. The performance reductions from the $20 \mathrm{~kg}$ barbell were likely driven by altered strategies of a deeper, slower, and less powerful countermovement that reduced the ability to utilize the stretch-shortening cycle. Further, the ability to use the stretch-shortening cycle and the jump performances were reduced across the fatiguing events for the unloaded and $10 \mathrm{~kg}$ loaded vest conditions. The loaded jumps with the $20 \mathrm{~kg}$ barbell were not affected as a result of the high-intensity military training/competition event. This may be due to the heavier $20 \mathrm{~kg}$ load being a test of strength, more than a test of power, which are typically more stable measures over brief intensive training bouts. Likewise, performing the CMJ with a shallower countermovement depth may result in a lower countermovement jump and, subsequently, more stable response to neuromuscular fatigue. Indices of the ability to utilize the stretch-shortening cycle and jump outcomes of CMJs, either unloaded or loaded up to $10 \mathrm{~kg}$, are useful to identify responses to neuromuscular fatigue within a bout of intensive training, which may be explained by alterations to jumping strategies (i.e., rate and magnitude of the countermovement). Loaded CMJs with a $20 \mathrm{~kg}$ barbell may be more conducive for measuring strength capabilities in tactical populations, which may be used to identify the ability to handle external loads for occupational requirements and indicate strength training adaptations, although continued research is necessary to confirm this hypothesis, especially over the military occupational specialties (MOS) of interest. To improve these outcomes, additional explosive plyometric training may be beneficial. Specifically, plyometric training with an emphasis on using a quick forceful countermovement should be employed across various loading conditions. Doing so may create a more sustainable force by improving the ability to handle external loading and resiliency to training-induced fatigue.

Author Contributions: Conceptualization, J.J.M., J.D.S., J.A.H. and A.G.T.; methodology, J.J.M., J.D.S., J.A.H. and A.G.T.; formal analysis, J.J.M.; investigation, J.D.S., J.R., J.A.H. and A.G.T.; resources, J.A.H. and A.G.T.; data curation, J.R. and A.G.T.; writing—original draft preparation, J.J.M.; writingreview and editing, J.D.S., J.R., J.A.H. and A.G.T.; visualization, J.J.M.; supervision, J.A.H. and A.G.T.; project administration, J.A.H. and A.G.T.; funding acquisition, J.A.H. and A.G.T. All authors have read and agreed to the published version of the manuscript.

Funding: This research was funded by the Office of Naval Research, contract number N00014-19-C-2042.

Institutional Review Board Statement: The study was conducted according to the guidelines of the Declaration of Helsinki and approved by the Institutional Review Board of West Virginia University $\# 1906605539$.

Informed Consent Statement: Informed consent was obtained from all subjects involved in the study.

Data Availability Statement: Data sharing is not applicable to this article.

Conflicts of Interest: The authors declare no conflict of interest.

\section{References}

1. Hauschild, V.D.; DeGroot, D.W.; Hall, S.M.; Grier, T.L.; Deaver, K.D.; Hauret, K.G.; Jones, B.H. Fitness tests and occupational tasks of Military interest: A systematic review of correlations. Occup. Environ. Med. 2017, 74, 144-153. [CrossRef]

2. Ruble, P.; Silverman, M.; Harrell, J.; Ringenberg, L.; Fruendt, J.; Walters, T.; Christiansen, L.; Llorente, M.; Barnett, S.D.; Scherb, B.; et al. Medical and physical readiness of the U.S. Army Reserve for Noble Eagle/Enduring Freedom/Iraqi Freedom: Recommendations for future mobilizations. Mil. Med. 2005, 170, 443-450. [CrossRef]

3. Bullock, S.H.; Jones, B.H.; Gilchrist, J.; Marshall, S.W. Prevention of physical training-related injuries: Recommendations for the Military and other active populations based on expedited systematic reviews. Am. J. Prev. Med. 2010, 38, S156-S181. [CrossRef] 
4. Maupin, D.; Schram, B.; Orr, R. Tracking training load and its implementation in tactical populations: A narrative review. Strength Cond. J. 2019, 41, 1-11. [CrossRef]

5. Knapik, J.J.; Graham, B.S.; Rieger, J.; Steelman, R.; Pendergrass, T. Activities associated with injuries in initial entry training. Mil. Med. 2013, 178, 500-506. [CrossRef]

6. Kaufman, K. Military training-related injuries surveillance, research, and prevention. Am. J. Prev. Med. 2000, 18, 54-63. [CrossRef]

7. Stannard, J.; Fortington, L. Musculoskeletal injury in Military Special Operations Forces: A systematic review. BMJ Mil. Health 2021. [CrossRef]

8. Knapik, J.J.; Reynolds, K. Load carriage-related injury mechanisms, risk factors, and prevention. In The Mechanobiology and Mechanophysiology of Military-Related Injuries; Gefen, A., Epstein, Y., Eds.; Studies in Mechanobiology, Tissue Engineering and Biomaterials; Springer International Publishing: Cham, Switzerland, 2016; pp. 107-137, ISBN 978-3-319-33012-9.

9. Orr, R.M.; Johnston, V.; Coyle, J.; Pope, R. Reported load carriage injuries of the Australian Army Soldier. J. Occup. Rehabil. 2015, 25, 316-322. [CrossRef] [PubMed]

10. Dijksma, C.I.; Bekkers, M.; Spek, B.; Lucas, C.; Stuiver, M. Epidemiology and financial burden of musculoskeletal injuries as the leading health problem in the Military. Mil. Med. 2020, 185, e480-e486. [CrossRef]

11. Andersen, K.A.; Grimshaw, P.N.; Kelso, R.M.; Bentley, D.J. Musculoskeletal lower limb injury risk in Army populations. Sports Med. Open 2016, 2, 22. [CrossRef]

12. Jensen, A.E.; Laird, M.; Jameson, J.T.; Kelly, K.R. Prevalence of musculoskeletal injuries sustained during Marine Corps Recruit Training. Mil. Med. 2019, 184, 511-520. [CrossRef]

13. Wardle, S.L.; Greeves, J.P. Mitigating the risk of musculoskeletal injury: A systematic review of the most effective injury prevention strategies for Military personnel. J. Sci. Med. Sport 2017, 20, S3-S10. [CrossRef]

14. Merrigan, J.J.; Stone, J.D.; Thompson, A.G.; Hornsby, W.G.; Hagen, J.A. Monitoring neuromuscular performance in Military personnel. Int. J. Environ. Res. Public Health 2020, 17, 9147. [CrossRef]

15. Fortes, M.B.; Diment, B.C.; Greeves, J.P.; Casey, A.; Izard, R.; Walsh, N.P. Effects of a daily mixed nutritional supplement on physical performance, body composition, and circulating anabolic hormones during 8 weeks of arduous Military training. Appl. Physiol. Nutr. Metab. 2011, 36, 967-975. [CrossRef] [PubMed]

16. Nindl, B.C.; Barnes, B.R.; Alemany, J.A.; Frykman, P.N.; Shippee, R.L.; Friedl, K.E. physiological consequences of U.S. Army Ranger Training. Med. Sci. Sports Exerc. 2007, 39, 1380-1387. [CrossRef] [PubMed]

17. Welsh, T.; Alemany, J.; Montain, S.; Frykman, P.; Tuckow, A.; Young, A.; Nindl, B. Effects of intensified Military field training on jumping performance. Int. J. Sports Med. 2008, 29, 45-52. [CrossRef] [PubMed]

18. Maupin, D.; Wills, T.; Orr, R.; Schram, B. Fitness profiles in elite tactical units: A critical review. Int. J. Exerc. Sci. 2018, 11, 1041-1062.

19. Joseph, A.; Wiley, A.; Orr, R.; Schram, B.; Dawes, J.J. The impact of load carriage on measures of power and agility in tactical occupations: A critical review. Int. J. Environ. Res. Public Health 2018, 15, 88. [CrossRef]

20. Orr, R.M.; Dawes, J.J.; Lockie, R.G.; Godeassi, D.P. The relationship between lower-body strength and power, and load carriage tasks: A critical review. Int. J. Exerc. Sci. 2019, 12, 1001-1022.

21. Šimenko, J.; Kovcan, B.; Pori, P.; Vodicar, J.; Vodicar, M.; Hadžic, V. The relationship between Army physical fitness and functional capacities in infantry members of the Slovenian Armed Forces. J. Strength Cond Res. 2020. [CrossRef]

22. Pihlainen, K.; Santtila, M.; Häkkinen, K.; Kyröläinen, H. Associations of physical fitness and body composition characteristics with simulated Military task performance. J. Strength Cond Res. 2018, 32, 1089-1098. [CrossRef] [PubMed]

23. Mala, J.; Szivak, T.K.; Flanagan, S.D.; Comstock, B.A.; Laferrier, J.Z.; Maresh, C.M.; Kraemer, W.J. The role of strength and power during performance of high intensity Military tasks under heavy load carriage. Us Army Med. Dep. J. 2015, 5-11.

24. Wu, P.P.-Y.; Sterkenburg, N.; Everett, K.; Chapman, D.W.; White, N.; Mengersen, K. Predicting fatigue using countermovement jump force-time signatures: PCA can distinguish neuromuscular versus metabolic fatigue. PLoS ONE 2019, 14, e0219295. [CrossRef] [PubMed]

25. Kennedy, R.; Drake, D. The Effect of acute fatigue on countermovement jump performance in rugby union players during preseason. J. Sports Med. Phys. Fit. 2017, 57, 1261-1266.

26. Domire, Z.J.; Challis, J.H. The influence of squat depth on maximal vertical jump performance. J. Sports Sci. 2007, 25, 193-200. [CrossRef]

27. Pérez-Castilla, A.; Rojas, F.J.; Gómez-Martínez, F.; García-Ramos, A. Vertical jump performance is affected by the velocity and depth of the countermovement. Sports Biomech. 2019, 1-16. [CrossRef]

28. Pérez-Castilla, A.; Weakley, J.; García-Pinillos, F.; Rojas, F.J.; García-Ramos, A. Influence of countermovement depth on the countermovement jump-derived reactive strength index modified. Eur. J. Sport Sci. 2020, 1-11. [CrossRef]

29. Sánchez-Sixto, A.; Harrison, A.; Floría, P. Larger Countermovement increases the jump height of countermovement jump. Sports 2018, 6, 131. [CrossRef]

30. Merrigan, J.J.; O’Toole, K.B.; Wutzke, C.J.; Jones, M.T. A kinetic and kinematic analysis of various drop jump performances in Army Reserve Officer Training Corps Cadets. J. Strength Cond Res. 2021, in press.

31. Jaworski, R.L.; Jensen, A.; Niederberger, B.; Congalton, R.; Kelly, K.R. Changes in combat task performance under increasing loads in active duty Marines. Mil. Med. 2015, 180, 179-186. [CrossRef] 
32. O'Neal, E.K.; Hornsby, J.H.; Kelleran, K.J. High-Intensity tasks with external load in Military applications: A review. Mil. Med. 2014, 179, 950-954. [CrossRef]

33. Orr, R.M.; Pope, R.; Johnston, V.; Coyle, J. Soldier occupational load carriage: A narrative review of associated injuries. Int. J. Inj. Contr. Saf. Promot. 2014, 21, 388-396. [CrossRef]

34. Kraska, J.M.; Ramsey, M.W.; Haff, G.G.; Fethke, N.; Sands, W.A.; Stone, M.E.; Stone, M.H. Relationship between strength characteristics and unweighted and weighted vertical jump height. Int. J. Sports Physiol. Perform. 2009, 4, 461-473. [CrossRef]

35. Brown, T.N.; O’Donovan, M.; Hasselquist, L.; Corner, B.; Schiffman, J.M. Lower limb flexion posture relates to energy absorption during drop landings with soldier-relevant body borne loads. Appl. Ergon. 2016, 52, 54-61. [CrossRef]

36. Tilbury-Davis, D.C.; Hooper, R.H. The Kinetic and Kinematic Effects of increasing load carriage upon the lower limb. Hum. Mov. Sci. 1999, 18, 693-700. [CrossRef]

37. McGinnis, R.S.; Cain, S.M.; Davidson, S.P.; Vitali, R.V.; Perkins, N.C.; McLean, S.G. Quantifying the effects of load carriage and fatigue under load on sacral kinematics during countermovement vertical jump with IMU-Based method. Sports Eng. 2016, 19, 21-34. [CrossRef]

38. HITT2019. Available online: https://usmc-mccs.org/hitt2019/ (accessed on 24 May 2021).

39. Heishman, A.D.; Daub, B.D.; Miller, R.M.; Freitas, E.D.S.; Frantz, B.A.; Bemben, M.G. Countermovement jump reliability performed with and without an arm swing in NCAA Division 1 intercollegiate basketball players. J. Strength Cond. Res. 2020, 34, 546-558. [CrossRef] [PubMed]

40. McMahon, J.J.; Suchomel, T.J.; Lake, J.P.; Comfort, P. Understanding the key phases of the countermovement jump force-time curve. Strength Cond. J. 2018, 40, 96-106. [CrossRef]

41. Chavda, S.; Bromley, T.; Jarvis, P.; Williams, S.; Bishop, C.; Turner, A.N.; Lake, J.P.; Mundy, P.D. Force-Time characteristics of the countermovement jump: Analyzing the curve in excel. Strength Cond. J. 2018, 40, 67-77. [CrossRef]

42. Merrigan, J.J.; Stone, J.D.; Hornsby, W.G.; Hagen, J.A. Identifying reliable and relatable force-time metrics in athletesconsiderations for the isometric mid-thigh pull and countermovement jump. Sports 2021, 9, 4. [CrossRef] [PubMed]

43. Kaiser, H.F.; Rice, J. Little Jiffy, Mark IV. Educ. Psychol. Meas. 1974, 34, 111-117. [CrossRef]

44. Cattell, R.B. The Scree Test for the Number of Factors. Multivar. Behav. Res. 1966, 1, 245-276. [CrossRef] [PubMed]

45. Kaiser, H.F. The application of electronic computers to factor analysis. Educ. Psychol. Meas. 1960, 20, 141-151. [CrossRef]

46. Abdi, H.; Williams, L.J. Principal Component Analysis. Wires Comput. Stat. 2010, 2, 433-459. [CrossRef]

47. Pinheiro, J.; Bates, D.; DebRoy, S.; Sarkar, D.; Team, R.C. Nlme: Linear and Nonlinear Mixed Effects Models. R Package Version 3.1-152. 2021. Available online: https:/ /CRAN.R-project.org/package=nlme (accessed on 20 April 2020).

48. Linthorne, N.P. The correlation between jump height and mechanical power in a countermovement jump is artificially inflated. Sports Biomech. 2021, 20, 3-21. [CrossRef]

49. McHugh, M.P.; Hickok, M.; Cohen, J.A.; Virgile, A.; Connolly, D.A.J. Is There a biomechanically efficient vertical ground reaction force profile for countermovement jumps? Transl. Sports Med. 2021, 4, 138-146. [CrossRef]

50. Gathercole, R.; Sporer, B.; Stellingwerff, T.; Sleivert, G. Alternative countermovement-jump analysis to quantify acute neuromuscular fatigue. Int. J. Sports Physiol. Perform. 2015, 10, 84-92. [CrossRef] [PubMed]

51. Ojanen, T.; Häkkinen, K.; Vasankari, T.; Kyröläinen, H. Changes in physical performance during $21 \mathrm{~d}$ of Military field training in warfighters. Mil. Med. 2018, 183, e174-e181. [CrossRef] [PubMed]

52. Nicol, C.; Avela, J.; Komi, P.V. The stretch-shortening cycle. Sports Med. 2006, 36, 977-999. [CrossRef]

53. Vaverka, F.; Jakubsova, Z.; Jandacka, D.; Zahradnik, D.; Farana, R.; Uchytil, J.; Supej, M.; Vodicar, J. The influence of an additional load on time and force changes in the ground reaction force during the countermovement vertical jump. J. Hum. Kinet. 2013, 38, 191-200. [CrossRef]

54. Norris, D.; Joyce, D.; Siegler, J.; Clock, J.; Lovell, R. Recovery of force-time characteristics after Australian rules football matches: Examining the utility of the isometric midthigh pull. Int. J. Sports Physiol. Perform. 2019, 14, 765-770. [CrossRef] [PubMed]

55. Swinton, P.A.; Stewart, A.D.; Lloyd, R.; Agouris, I.; Keogh, J.W.L. Effect of load positioning on the kinematics and kinetics of weighted vertical jumps. J. Strength Cond. Res. 2012, 26, 906-913. [CrossRef] [PubMed] 